\title{
The Lee-Wick standard model
}

\author{
Benjamín Grinstein, ${ }^{1, *}$ Donal O'Connell, ${ }^{2, \dagger}$ and Mark B. Wise ${ }^{2, *}$ \\ ${ }^{1}$ Department of Physics, University of California at San Diego, La Jolla, California 92093, USA \\ ${ }^{2}$ California Institute of Technology, Pasadena, California 91125, USA
}

(Received 24 July 2007; published 14 January 2008)

\begin{abstract}
We construct a modification of the standard model which stabilizes the Higgs mass against quadratically divergent radiative corrections, using ideas originally discussed by Lee and Wick in the context of a finite theory of quantum electrodynamics. The Lagrangian includes new higher derivative operators. We show that the higher derivative terms can be eliminated by introducing a set of auxiliary fields; this allows for convenient computation and makes the physical interpretation more transparent. The theory is thought to be unitary, but nevertheless, it does not satisfy the usual analyticity conditions.
\end{abstract}

DOI: 10.1103/PhysRevD.77.025012

PACS numbers: $12.60 . \mathrm{Cn}, 14.80 . \mathrm{Cp}$

\section{INTRODUCTION}

The extreme fine-tuning needed to keep the Higgs mass small compared to the Planck scale (i.e., the hierarchy puzzle) has motivated many extensions of the minimal standard model. All of these contain new physics, beyond that in the minimal standard model, which might be observed at the Large Hadron Collider (LHC). The most widely explored of these extensions is low energy supersymmetry. In this paper we introduce another extension of the standard model that solves the hierarchy puzzle.

Our approach builds on the work of Lee and Wick $[1,2]$ who studied the possibility that the regulator propagator in Pauli-Villars corresponds to a physical degree of freedom. Quantum electrodynamics with a photon propagator that includes the regulator term is a higher derivative version of QED. The higher derivative propagator contains two poles, one corresponding to the massless photon, and the other corresponding to a massive Lee-Wick photon (LW photon). A problem with this approach is that the residue of the massive LW-photon pole has the wrong sign. Classically, this sign indicates an instability of the theory. At the quantum level, the sign is associated with a nonpositive definite norm on the Hilbert space; consequently, there are zero- and negative-norm states in the Hilbert space. Lee and Wick argued that one can make physical sense of such a theory. In order to preserve unitarity, there must be a mechanism which removes all states containing $n \geq 1$ Lee-Wick photons from the Hilbert space. Since the LW photon is massive, it develops a width, and consequently states containing one LW photon do not appear in the Hilbert space. However, cuts corresponding to two or more LW photons going on shell can still occur in Feynman graphs. Lee and Wick proposed a modification of the usual integration contour in Feynman diagrams to remove these states at low orders in perturbation theory.

\footnotetext{
*bgrinstein@ucsd.edu

†donal@ theory.caltech.edu

ॠwise@ theory.caltech.edu
}

This proposal was later improved by Cutkosky et al. [3]. ${ }^{1}$ There is no general proof of unitarity at arbitrary loop order for Lee-Wick theories; however, there is no known example of unitarity violation. For further discussion of these issues, see [7]. In order to remove the classical instability of the theory, Lee and Wick proposed that a future boundary condition should be imposed on the theory. ${ }^{2}$ The presence of this boundary condition leads to a modification in the usual relationship between the $S$ matrix and the time evolution operator, as well as an unusual time ordering of events, as discussed in [7].

An attempt was made to find a path integral formulation of Lee-Wick theories in [9]; however, this attempt was unsuccessful. Scalar Lee-Wick theories, including the Higgs sector of the standard model, were studied in [10] using large $N$ and lattice methods. The results of this work are encouraging; for example, the theory was seen to be unitary at the nonperturbative level. In the context of higher derivative gravity, Antoniadis and Tomboulis [11] have argued that the theory must be unitarity since the mass associated with the ghost pole is gauge dependent.

The theory of QED that Lee and Wick studied is finite. In this paper we propose to extend their idea to the standard model, removing the quadratic divergence associated with the Higgs mass, and thus solving the hierarchy problem. ${ }^{3}$ In the LW-standard model, every field in the minimal standard model has a higher derivative kinetic term that introduces a corresponding massive LW resonance. These masses are additional free parameters in the theory and must be high enough to evade current experimental constraints. For the non-Abelian gauge bosons the higher derivative kinetic term has, because of gauge invariance,

\footnotetext{
${ }^{1}$ The consistency of this approach is controversial, and has been discussed in [4-6].

${ }^{2}$ From the point of view of the Euclidean path integral, it has been argued that such boundary conditions are natural [8].

${ }^{3}$ Previously, it was observed that the electroweak sector of the standard model could be extended to include additional scalars which remove the divergences in leptonic self-masses at oneloop order [12].
} 
new higher derivative interactions. Hence the resulting theory is not finite; however, we argue that it does not give rise to a quadratic divergence in the Higgs mass, and so solves the hierarchy puzzle. A power counting argument and some explicit one-loop calculations are given to demonstrate this. For explicit calculations, and to make the physics clearer, it is useful to remove the higher derivative terms in the Lagrangian density by introducing auxiliary LW fields that, when integrated out, reproduce the higher derivative terms in the action.

The LW-standard model ${ }^{4}$ has a new parameter for each standard model field, which corresponds physically to the tree-level mass of its LW-partner resonance. Explicit calculations can be performed in this theory at any order in perturbation theory, and the experimental consequences for physics at the LHC, and elsewhere, can be studied. LeeWick theories are unusual; however, even if one does not take the particular model we present as the correct theory of nature at the $\mathrm{TeV}$ scale our work does suggest that a further examination of higher derivative theories is warranted. Some previous work on field theories with nonlocal actions that contain terms with an infinite number of derivatives can be found in Ref. [13].

\section{A TOY MODEL}

To illustrate the physics of Lee-Wick theory $[1,2,9,10]$ in a simple setting, we consider in this section a theory of one self-interacting scalar field, $\hat{\phi}$, with a higher derivative term. The Lagrangian density is

$$
\mathcal{L}_{\text {hd }}=\frac{1}{2} \partial_{\mu} \hat{\phi} \partial^{\mu} \hat{\phi}-\frac{1}{2 M^{2}}\left(\partial^{2} \hat{\phi}\right)^{2}-\frac{1}{2} m^{2} \hat{\phi}^{2}-\frac{1}{3 !} g \hat{\phi}^{3},
$$

so the propagator of $\hat{\phi}$ in momentum space is given by

$$
\hat{D}(p)=\frac{i}{p^{2}-p^{4} / M^{2}-m^{2}} .
$$

For $M \gg m$, this propagator has poles at $p^{2} \simeq m^{2}$ and also at $p^{2} \simeq M^{2}$. Thus, the propagator describes more than one degree of freedom.

We can make these new degrees of freedom manifest in the Lagrangian density in a simple way. First, let us introduce an auxiliary scalar field $\tilde{\phi}$, so that we can write the theory as

$$
\begin{aligned}
\mathcal{L}= & \frac{1}{2} \partial_{\mu} \hat{\phi} \partial^{\mu} \hat{\phi}-\frac{1}{2} m^{2} \hat{\phi}^{2}-\tilde{\phi} \partial^{2} \hat{\phi}+\frac{1}{2} M^{2} \tilde{\phi}^{2} \\
& -\frac{1}{3 !} g \hat{\phi}^{3} .
\end{aligned}
$$

Since $\mathcal{L}$ is quadratic in $\tilde{\phi}$, the equations of motion of $\tilde{\phi}$ are exact at the quantum level. Removing $\tilde{\phi}$ from $\mathcal{L}$ with their equations of motion reproduces $\mathcal{L}_{\text {hd }}$ in Eq. (1).

${ }^{4} \mathrm{LW}$ extension of the standard model would be more precise.
Next, we define $\phi=\hat{\phi}+\tilde{\phi}$. In terms of this variable, the Lagrangian in Eq. (3) becomes, after integrating by parts,

$$
\begin{aligned}
\mathcal{L}= & \frac{1}{2} \partial_{\mu} \phi \partial^{\mu} \phi-\frac{1}{2} \partial_{\mu} \tilde{\phi} \partial^{\mu} \tilde{\phi}+\frac{1}{2} M^{2} \tilde{\phi}^{2} \\
& -\frac{1}{2} m^{2}(\phi-\tilde{\phi})^{2}-\frac{1}{3 !} g(\phi-\tilde{\phi})^{3} .
\end{aligned}
$$

In this form, it is clear that there are two kinds of scalar field: a normal scalar field $\phi$ and a new field $\tilde{\phi}$, which we will refer to as a LW field. The sign of the quadratic Lagrangian of the LW field is opposite to the usual sign so one may worry about stability of the theory, even at the classical level. We will return to this point. If we neglect the mass $m$ for simplicity, the propagator of $\tilde{\phi}$ is given by

$$
\tilde{D}(p)=\frac{-i}{p^{2}-M^{2}} .
$$

The LW field is associated with a nonpositive definite norm on the Hilbert space, as indicated by the unusual sign of its propagator. Consequently, if this state were to be stable, unitarity of the $S$ matrix would be violated. However, as emphasized by Lee and Wick, unitarity can be preserved if $\tilde{\phi}$ decays. This occurs in the theory described by Eq. (4) because $\tilde{\phi}$ is heavy and can decay to two $\phi$-particles.

In the presence of the mass $m$, there is mixing between the scalar field $\phi$ and the LW scalar $\tilde{\phi}$. We can diagonalize this mixing without spoiling the diagonal form of the derivative terms by performing a symplectic rotation on the fields:

$$
\left(\begin{array}{c}
\phi \\
\tilde{\phi}
\end{array}\right)=\left(\begin{array}{cc}
\cosh \theta & \sinh \theta \\
\sinh \theta & \cosh \theta
\end{array}\right)\left(\begin{array}{c}
\phi^{\prime} \\
\tilde{\phi}^{\prime}
\end{array}\right) .
$$

This transformation diagonalizes the Lagrangian if

$$
\tanh 2 \theta=\frac{-2 m^{2} / M^{2}}{1-2 m^{2} / M^{2}} .
$$

A solution for the angle $\theta$ exists provided $M>2 m$. The Lagrangian (4) describing the system becomes

$$
\begin{aligned}
\mathcal{L}= & \frac{1}{2} \partial_{\mu} \phi^{\prime} \partial^{\mu} \phi^{\prime}-\frac{1}{2} m^{\prime 2} \phi^{\prime 2}-\frac{1}{2} \partial_{\mu} \tilde{\phi}^{\prime} \partial^{\mu} \tilde{\phi}^{\prime}+\frac{1}{2} M^{\prime 2} \tilde{\phi}^{\prime 2} \\
& -\frac{1}{3 !}(\cosh \theta-\sinh \theta)^{3} g\left(\phi^{\prime}-\tilde{\phi}^{\prime}\right)^{3},
\end{aligned}
$$

where $m^{\prime}$ and $M^{\prime}$ are the masses of the diagonalized fields. Notice the form of the interaction; we can define $g^{\prime}=$ $(\cosh \theta-\sinh \theta)^{3} g$ and then drop the primes to obtain a convenient Lagrangian for computation. ${ }^{5}$

Introducing the LW fields makes the physics of the theory clear. There are two fields; the heavy LW scalar decays to the lighter scalar. At loop level, the presence of

\footnotetext{
${ }^{5}$ In the following, we will always assume that $M \gg m$ so that $g^{\prime} \simeq g$.
} 
the heavier scalar improves the convergence of loop graphs at high energy consistent with our expectations from the higher derivative form of the theory. We can use the familiar technology of perturbative quantum field theory (appropriately modified [3]) to compute quantum corrections to the physics.

It is worth pausing for a moment to consider loop corrections to the two point function of the LW field. Using the one-loop self-energy, the full propagator for the LW scalar is given, near $p^{2}=M^{2}$, by

$$
\begin{aligned}
\tilde{D}(p) & =\frac{-i}{p^{2}-M^{2}}+\frac{-i}{p^{2}-M^{2}}\left(-i \Sigma\left(p^{2}\right)\right) \frac{-i}{p^{2}-M^{2}}+\cdots \\
& =\frac{-i}{p^{2}-M^{2}+\Sigma\left(p^{2}\right)} .
\end{aligned}
$$

Note that, unlike for ordinary scalars, there is a plus sign in front of the self-energy $\Sigma\left(p^{2}\right)$ in the denominator. This sign is significant; for example, if one defines the width in the usual way (i.e., near the pole the propagator has denominator $\left.p^{2}-M^{2}+i M \Gamma\right)$ then, from a one-loop computation of the self-energy $\Sigma$, the width of the LW field is (for $\operatorname{Im} p^{2}>0$ )

$$
\Gamma=-\frac{g^{2}}{32 \pi M} \sqrt{1-\frac{4 m^{2}}{M^{2}}} .
$$

This width differs in sign from widths of the usual particles we encounter. With this result in hand, we can demonstrate how unitarity of the theory is maintained in an explicit example. Consider $\phi \phi$ scattering in this theory. From unitarity, the imaginary part of the forward scattering amplitude, $\mathcal{M}$, must be a positive quantity. Near $p^{2}=$ $M^{2}$, the scattering is dominated by the $\tilde{\phi}$ pole and therefore the imaginary part of the amplitude is given for $\operatorname{Im} p^{2}>0$ by

$$
\operatorname{Im} \mathcal{M}=-g^{2} \frac{M \Gamma}{\left(p^{2}-M^{2}\right)^{2}+M^{2} \Gamma^{2}} .
$$

The unusual sign of the propagator is compensated by the unusual sign of the decay width.

As another consequence of this sign, the poles associated with these LW particles occur on the physical sheet of the analytic continuation of the $S$ matrix, in violation of the usual rules of $S$ matrix theory. These signs are also associated with exponential growth of disturbances, which is related to the stability concerns alluded to earlier. Lee and Wick, and Cutkowsky et al. argued that one can nevertheless make sense of these theories by modifying the usual contour prescription for momentum integrals. The Feynman $i \epsilon$ prescription can be thought of as a deformation of the contour such that the poles on the real axis are appropriately above or below the contour. The Lee-Wick prescription is equivalent to imposing the boundary condition that there are no outgoing exponentially growing modes. It is well known that such future boundary con- ditions cause violations of causality. In the Lee-Wick theory the acausal effects occur only on microscopic scales, and show up as a peculiar time ordering of events; for example, the decay products of a Lee-Wick particle appear at times before the Lee-Wick particle itself is created. It is believed that this theory does not produce violations of causality, or any paradoxes, on a macroscopic scale [7].

\section{THE HIERARCHY PROBLEM AND LEE-WICK THEORY}

In this section, we consider a scalar in the fundamental representation interacting with gauge bosons. We find the Lagrange density for the LW version of such a theory and show by power counting appropriate to the higher derivative version of the theory that the scalar mass is free of quadratic divergences. We then show by an explicit oneloop calculation that the ordinary scalar and the massive LW fields do not receive a quadratically divergent contribution to their pole masses.

\section{A. Gauge fields}

The higher derivative Lagrangian in the gauge sector is

$$
\mathcal{L}_{\text {hd }}=-\frac{1}{2} \operatorname{tr} \hat{F}_{\mu \nu} \hat{F}^{\mu \nu}+\frac{1}{M_{A}^{2}} \operatorname{tr}\left(\hat{D}^{\mu} \hat{F}_{\mu \nu}\right)\left(\hat{D}^{\lambda} \hat{F}_{\lambda}{ }^{\nu}\right),
$$

where $\hat{F}_{\mu \nu}=\partial_{\mu} \hat{A}_{\nu}-\partial_{\nu} \hat{A}_{\mu}-i g\left[\hat{A}_{\mu}, \hat{A}_{\nu}\right]$, and $\hat{A}_{\mu}=$ $\hat{A}_{\mu}^{A} T^{A}$ with $T^{A}$ the generators of the gauge group $G$ in the fundamental representation. We can now eliminate the higher derivative term by introducing auxiliary massive gauge bosons $\tilde{A}$. Each gauge boson is described by a Lagrangian

$$
\mathcal{L}=-\frac{1}{2} \operatorname{tr} \hat{F}_{\mu \nu} \hat{F}^{\mu \nu}-M_{A}^{2} \operatorname{tr} \tilde{A}_{\mu} \tilde{A}^{\mu}+2 \operatorname{tr} \hat{F}_{\mu \nu} \hat{D}^{\mu} \tilde{A}^{\nu},
$$

where $\hat{D}_{\mu} \tilde{A}_{\nu}=\partial_{\mu} \tilde{A}_{\nu}-i g\left[\hat{A}_{\mu}, \tilde{A}_{\nu}\right]$. To diagonalize the kinetic terms, we introduce shifted fields defined by

$$
\hat{A}_{\mu}=A_{\mu}+\tilde{A}_{\mu} \text {. }
$$

The Lagrangian becomes

$$
\begin{aligned}
\mathcal{L}= & -\frac{1}{2} \operatorname{tr} F_{\mu \nu} F^{\mu \nu}+\frac{1}{2} \operatorname{tr}\left(D_{\mu} \tilde{A}_{\nu}-D_{\nu} \tilde{A}_{\mu}\right)\left(D^{\mu} \tilde{A}^{\nu}-D^{\nu} \tilde{A}^{\mu}\right) \\
& -i g \operatorname{tr}\left(\left[\tilde{A}_{\mu}, \tilde{A}_{\nu}\right] F^{\mu \nu}\right)-\frac{3}{2} g^{2} \operatorname{tr}\left(\left[\tilde{A}_{\mu}, \tilde{A}_{\nu}\right]\left[\tilde{A}^{\mu}, \tilde{A}^{\nu}\right]\right) \\
& -4 i g \operatorname{tr}\left(\left[\tilde{A}_{\mu}, \tilde{A}_{\nu}\right] D^{\mu} \tilde{A}^{\nu}\right)-M_{A}^{2} \operatorname{tr}\left(\tilde{A}_{\mu} \tilde{A}^{\mu}\right) .
\end{aligned}
$$

Note that for a $U(1)$ gauge boson all the commutators vanish, there are no traces and an extra overall factor of $1 / 2$.

To perform perturbative calculations, we must introduce a gauge fixing term. We could introduce such a term in the higher derivative Lagrangian, Eq. (12), in terms of the Lagrangian involving $A$ and $\tilde{A}$, Eq. (15), or even in the Lagrangian with mixed kinetic terms for $\hat{A}$ and $\tilde{A}$, Eq. (13). 
As is usual in gauge theories, all of these choices will yield the same results for physical quantities, but they may differ for unphysical quantities. Different gauge choices can differ on how divergent unphysical quantities are. Therefore, we will only compute physical pole masses below. In these computations, we introduce a covariant gauge fixing term for the gauge bosons, $A_{\mu}^{A}$, in the two field description of the theory given in Eq. (15). In this choice of gauge, the propagator for the gauge bosons is given by

$$
D_{\mu \nu}^{A B}(p)=-\delta^{A B} \frac{i}{p^{2}}\left(\eta_{\mu \nu}-(1-\xi) \frac{p_{\mu} p_{\nu}}{p^{2}}\right),
$$

while the propagator for the LW-gauge field is

$$
\tilde{D}_{\mu \nu}^{A B}(p)=\delta^{A B} \frac{i}{p^{2}-M_{A}^{2}}\left(\eta_{\mu \nu}-\frac{p_{\mu} p_{\nu}}{M_{A}^{2}}\right) .
$$

\section{B. Scalar matter}

Let us move on to consider scalar matter transforming in the fundamental representation of the gauge group. In ordinary field theory, such a scalar field has a quadratic divergence in its pole mass. The higher derivative Lagrangian is given in terms of the scalar field $\hat{\phi}$ by

$$
\begin{aligned}
\mathcal{L}_{\mathrm{hd}}= & \left(\hat{D}_{\mu} \hat{\phi}\right)^{\dagger}\left(\hat{D}^{\mu} \hat{\phi}\right)-\frac{1}{M_{\phi}^{2}}\left(\hat{D}_{\mu} \hat{D}^{\mu} \hat{\phi}\right)^{\dagger}\left(\hat{D}_{\nu} \hat{D}^{\nu} \hat{\phi}\right) \\
& -V(\hat{\phi}) .
\end{aligned}
$$

We eliminate the higher derivative term by introducing a $\mathrm{LW}$-scalar multiplet $\tilde{\phi}$. Then the Lagrangian is given in terms of the two fields $\hat{\phi}$ and $\tilde{\phi}$ by

$$
\begin{aligned}
\mathcal{L}= & \left(\hat{D}_{\mu} \hat{\phi}\right)^{\dagger}\left(\hat{D}^{\mu} \hat{\phi}\right)+M_{\phi}^{2} \tilde{\phi}^{\dagger} \tilde{\phi}+\left(\hat{D}_{\mu} \hat{\phi}\right)^{\dagger}\left(\hat{D}^{\mu} \tilde{\phi}\right) \\
& +\left(\hat{D}^{\mu} \tilde{\phi}\right)^{\dagger}\left(\hat{D}^{\mu} \hat{\phi}\right)-V(\hat{\phi}),
\end{aligned}
$$

where the covariant derivative is

$$
\hat{D}_{\mu}=\partial_{\mu}+i g \hat{A}_{\mu}^{A} T^{A} .
$$

For simplicity we take the ordinary scalar to have no potential at tree level, $V(\hat{\phi})=0$. It is not hard to include a potential for $\hat{\phi}$ in the analysis, and to show that the potential does not change our results.

We diagonalized the pure gauge sector by shifting the gauge fields; in terms of the shifted gauge fields the hatted covariant derivative is

$$
\hat{D}_{\mu}=D_{\mu}+i g \tilde{A}_{\mu}^{A} T^{A},
$$

where $D_{\mu}=\partial_{\mu}+i g A_{\mu}^{A} T^{A}$ is the usual covariant derivative. To diagonalize the scalar kinetic terms, we again shift the field

$$
\hat{\phi}=\phi-\tilde{\phi} .
$$

The scalar Lagrangian becomes

$$
\begin{aligned}
\mathcal{L}= & \left(D_{\mu} \phi\right)^{\dagger} D^{\mu} \phi-\left(D_{\mu} \tilde{\phi}\right)^{\dagger} D^{\mu} \tilde{\phi}+M_{\phi}^{2} \tilde{\phi}^{\dagger} \tilde{\phi} \\
& +i g\left(D^{\mu} \phi\right)^{\dagger} \tilde{A}_{\mu}^{A} T^{A} \phi+g^{2} \phi^{\dagger} \tilde{A}_{\mu}^{A} T^{A} \tilde{A}^{B \mu} T^{B} \phi \\
& -i g \phi^{\dagger} \tilde{A}_{\mu}^{A} T^{A} D^{\mu} \phi-i g\left(D^{\mu} \tilde{\phi}\right)^{\dagger} \tilde{A}_{\mu}^{A} T^{A} \tilde{\phi} \\
& +i g \tilde{\phi}^{\dagger} \tilde{A}_{\mu}^{A} T^{A} D^{\mu} \tilde{\phi}-g^{2} \tilde{\phi}^{\dagger} \tilde{A}_{\mu}^{A} T^{A} \tilde{A}^{B \mu} T^{B} \tilde{\phi}
\end{aligned}
$$

\section{Power counting}

Having defined the higher derivative and LW forms of the theory, we present a power counting argument for the higher derivative version of the theory which indicates that the only physical divergences in the theory are logarithmic. Since the power counting argument depends on the behavior of Feynman graphs at high energies, we only need to consider the terms in the Lagrangian which are most important at high energies.

For the perturbative power counting argument in the higher derivative theory, it is necessary to fix the gauge. We choose to add a covariant gauge fixing term $-\left(\partial_{\mu} \hat{A}^{A \mu}\right)^{2} / 2 \xi$ to the Lagrange density and introduce Faddeev-Popov ghosts that couple to the gauge bosons in the usual way. Then the propagator for the gauge field is

$$
\hat{D}_{\mu \nu}^{A B}(p)=\delta^{A B} \frac{-i}{p^{2}-p^{4} / M_{A}^{2}}\left(\eta_{\mu \nu}-(1-\xi) \frac{p_{\mu} p_{\nu}}{p^{2}}-\xi \frac{p_{\mu} p_{\nu}}{M_{A}^{2}}\right) .
$$

We work in $\xi=0$ gauge. Note that $\xi=0$ corresponds to Landau gauge and that the gauge boson propagator scales as $p^{-4}$ at high energy. The propagator for the scalar in the fundamental representation is

$$
\hat{D}^{a b}(p)=\delta^{a b} \frac{i}{p^{2}-p^{4} / M_{\phi}^{2}} .
$$

At large momenta the scalar propagator scales as $p^{-4}$ while the Faddeev-Popov ghost propagator scales as $p^{-2}$, as usual. There are three kinds of vertices: those where only gauge bosons interact, vertices where gauge bosons interact with two scalars, and vertices where two ghosts interact with one gauge boson. A vertex where $n$ vectors interact (with no scalars) scales as $p^{6-n}$ while a vertex with two scalars and $n$ vectors scales as $p^{4-n}$. The vertex between two ghosts and one gauge field scales as one power of $p$, as usual.

Consider an arbitrary Feynman graph with $L$ loops, $I^{\prime}$ internal vector lines, $I$ internal scalar lines, $I_{g}$ internal ghost lines, and with $V_{n}^{\prime}$ or $V_{n}$ vertices with $n$ vectors and zero or two scalar particles, respectively. We also suppose there are $V_{g}$ ghost vertices. Then the superficial degree of divergence, $d$, is 


$$
d=4 L-4 I^{\prime}-4 I-2 I_{g}+\sum_{n} V_{n}^{\prime}(6-n)+\sum_{n} V_{n}(4-n)+V_{g} .
$$

We can simplify this expression using some identities. First, the number of loops is related to the total number of propagators and vertices by

$$
L=I+I^{\prime}+I_{g}-\sum_{n}\left(V_{n}^{\prime}+V_{n}\right)-V_{g}+1,
$$

while the total number of lines entering or leaving the vertices is related to the number of propagators and external lines by

$$
\begin{aligned}
& \sum_{n}\left(n V_{n}^{\prime}+(n+2) V_{n}\right)+3 V_{g} \\
& =2\left(I+I^{\prime}+I_{g}\right)+E+E^{\prime}+E_{g},
\end{aligned}
$$

where $E$ is the number of external scalars, $E^{\prime}$ is the number of external vectors, and $E_{g}$ is the number of external ghosts. Finally, because the Lagrangian is quadratic in the number of scalars and ghosts, the number of scalar lines and ghost lines is separately conserved. Thus,

$$
2 \sum_{n} V_{n}=2 I+E, \quad 2 V_{g}=2 I_{g}+E_{g} .
$$

With these identities in hand, we may express the superficial degree of divergence as

$$
d=6-2 L-E-E^{\prime}-2 E_{g} .
$$

Gauge invariance removes the potential quadratic divergence in the gauge boson two point function. Scalar mass renormalizations have $E=2$, so that $d=4-2 L$. Consequently, the only possible quadratic divergence in the scalar mass is at one loop. However, gauge invariance also removes the divergence in the scalar mass renormalization, because two of the derivatives must act on the external legs. To see this, note that the interaction involves

$$
\phi^{\dagger} D^{4} \phi \sim \phi^{\dagger}\left(\partial^{2}+\partial \cdot A+A \cdot \partial+A^{2}\right)^{2} \phi .
$$

Since we are working in Lorentz gauge, $\partial \cdot A=0$. We may ignore the $A^{2}$ term compared to the $A \cdot \partial$ term, as it is less divergent. Thus the most divergent terms in the interaction are $\phi^{\dagger} A \cdot \partial \partial^{2} \phi$ or $\phi^{\dagger} \partial^{2} A \cdot \partial \phi$, where the $\phi$ acted on by the derivatives is an internal line. But by integration by parts and use of the gauge condition, we see that, at one loop, we can always take one of the derivatives to act on the external scalar. Thus the theory at hand is at most logarithmically divergent. ${ }^{6}$

\footnotetext{
${ }^{6}$ It may seem that adding operators with more than four derivatives could yield a finite theory, but that is not the case. These theories are still logarithmically divergent.
}

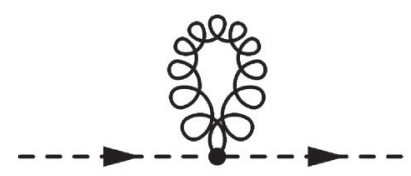

(a)

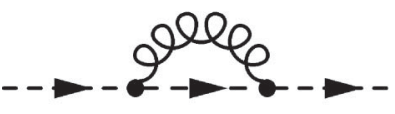

(c)

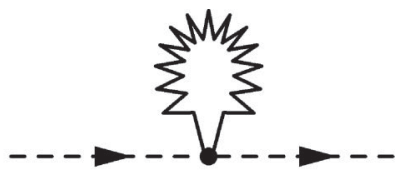

(b)

(d)

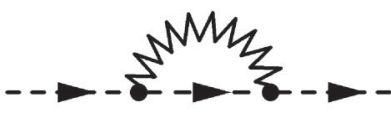

FIG. 1. One-loop mass renormalization of the normal scalar field. The curly line is a gauge field while the zigzag line is the LW-gauge field. The dashed line represents the scalar field.

\section{One-loop pole mass}

The power counting argument above was presented in the higher derivative version of the theory. As a check of the formalism we show, in the LW version of the theory, that the shift in the pole masses of the ordinary scalar, the LW scalar, and the LW-gauge boson do not receive quadratically divergent contributions at one loop. It is important to compute a physical quantity since it is for these that the higher derivative theory and the theory with LW fields give equivalent results. ${ }^{7}$ We perform the computations in Feynman gauge, using the propagators in Eqs. (16) and (17), and regulate our diagrams where necessary using dimensional regularization with dimension $n$.

\section{The normal scalar}

At one loop, there are four graphs contributing to the scalar mass, as shown in Fig. 1. We find

$$
\begin{aligned}
& -i \Sigma_{a}(0)=g^{2} C_{2}(N) \int \frac{d^{n} k}{(2 \pi)^{n}} \frac{n}{k^{2}} \\
& -i \Sigma_{b}(0)=-g^{2} C_{2}(N) \int \frac{d^{n} k}{(2 \pi)^{n}}\left(\frac{n-1}{k^{2}-M_{A}^{2}}-\frac{1}{M_{A}^{2}}\right) \\
& -i \Sigma_{c}(0)=-g^{2} C_{2}(N) \int \frac{d^{n} k}{(2 \pi)^{n}} \frac{1}{k^{2}} \\
& -i \Sigma_{d}(0)=-g^{2} C_{2}(N) \int \frac{d^{n} k}{(2 \pi)^{n}} \frac{1}{M_{A}^{2}}
\end{aligned}
$$

We see that the quartic and quadratic divergences in these expressions cancel in the sum, so that the mass is only logarithmically divergent.

\footnotetext{
${ }^{7}$ We have fixed different gauges in our discussion of the power counting argument in the higher derivative theory and our explicit computations in the LW version of the theory. Consequently, we can only expect agreement between these theories for physical quantities.
} 


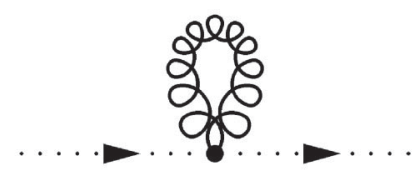

(a)

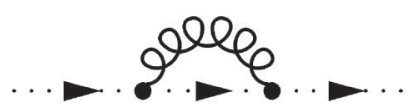

(c)

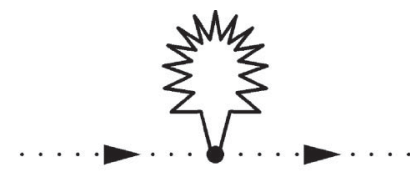

(b)

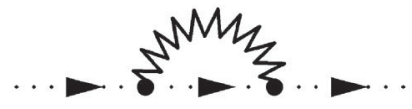

(d)
FIG. 2. One-loop mass renormalization of the LW-scalar field. The dotted line represents the LW-scalar field while the other propagators are as in Fig. 1.

\section{The LW scalar}

At one loop the shift in the pole mass is determined by the self-energy $\Sigma\left(p^{2}\right)$ evaluated at $p^{2}=M_{\phi}^{2}$. The Feynman graphs are shown in Fig. 2. We find

$$
\begin{aligned}
-i \Sigma_{a}\left(M_{\phi}^{2}\right)= & -g^{2} C_{2}(N) \int \frac{d^{n} k}{(2 \pi)^{n}} \frac{n}{k^{2}} \\
-i \Sigma_{b}\left(M_{\phi}^{2}\right)= & g^{2} C_{2}(N) \int \frac{d^{n} k}{(2 \pi)^{n}}\left(\frac{n-1}{k^{2}-M_{A}^{2}}-\frac{1}{M_{A}^{2}}\right) \\
-i \Sigma_{c}\left(M_{\phi}^{2}\right)= & g^{2} C_{2}(N) \int \frac{d^{n} k}{(2 \pi)^{n}} \\
& \times\left(\frac{1}{k^{2}-2 p \cdot k}+\frac{4 M_{\phi}^{2}-4 p \cdot k}{k^{2}\left(k^{2}-2 p \cdot k\right)}\right) \\
-i \Sigma_{d}\left(M_{\phi}^{2}\right)= & g^{2} C_{2}(N) \int \frac{d^{n} k}{(2 \pi)^{n}} \\
& \times\left(\frac{1}{M_{A}^{2}}-\frac{4 M_{\phi}^{2}-2 p \cdot k}{\left(k^{2}-M_{A}^{2}\right)\left(k^{2}-2 p \cdot k\right)}\right)
\end{aligned}
$$

Once again, the quartic and quadratic divergence cancel in the sum of the graphs, so that there is only a logarithmic divergence in the mass of the LW scalar.

\section{The $L W$ vector}

For the LW vectors the self-energy tensor has the form

$$
\Sigma_{\mu \nu}^{A B}\left(p^{2}\right)=\delta^{A B}\left[\Sigma\left(p^{2}\right) \eta_{\mu \nu}+\Sigma^{\prime}\left(p^{2}\right) p_{\mu} p_{\nu}\right]
$$

The shift in the pole mass is determined by $\Sigma\left(M_{A}^{2}\right)$. The relevant graphs are shown in Fig. 3. They are very divergent. There are individual terms in Fig. 3(c) that diverge as the sixth power of a momentum cutoff. However these cancel. There is also a quartic divergence in diagrams (b), (c), and (d) that cancels between them. To check that the quadratic divergence cancels we regulate the diagrams with dimensional regularization. In $n$ dimensions, a quadratic divergence manifests itself as a pole at $n=2$. Hence, we set $n=2-\epsilon$, expand about $\epsilon=0$, and extract the $1 / \epsilon$ part of $\Sigma\left(M_{A}^{2}\right)$. We find that (a)

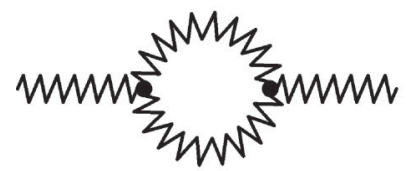

(c)

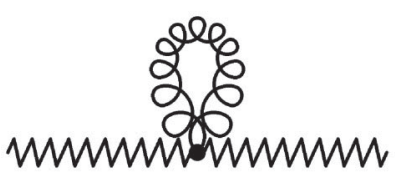

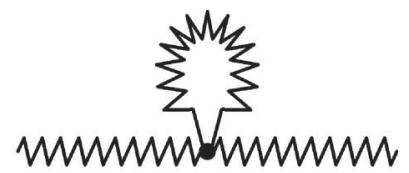

(b)

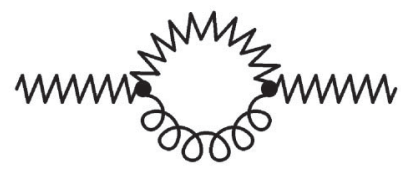

(d)
FIG. 3. One-loop mass renormalization of the LW-vector field. The propagators are as in Fig. 1.

$$
\begin{aligned}
& -i \Sigma_{a}\left(M_{A}^{2}\right)=\frac{i g^{2}}{4 \pi} C_{2}(G)\left(-\frac{2}{\epsilon}\right), \\
& -i \Sigma_{b}\left(M_{A}^{2}\right)=\frac{i g^{2}}{4 \pi} C_{2}(G)\left(\frac{3}{\epsilon}\right), \\
& -i \Sigma_{c}\left(M_{A}^{2}\right)=\frac{i g^{2}}{4 \pi} C_{2}(G)\left(-\frac{6}{\epsilon}\right), \\
& -i \Sigma_{d}\left(M_{A}^{2}\right)=\frac{i g^{2}}{4 \pi} C_{2}(G)\left(\frac{5}{\epsilon}\right) .
\end{aligned}
$$

As expected, the $1 / \epsilon$ pole cancels in the sum. Finally, we note that there are quadratic divergences in $\Sigma_{\mu \nu}^{A B}\left(p^{2}\right)$. Only the gauge invariant physical quantity $\Sigma\left(M_{A}^{2}\right)$ must be free of quadratic divergences.

\section{LEE-WICK STANDARD MODEL LAGRANGIAN}

Now that we have understood why the radiative correction to the Higgs mass cancels in these higher derivative theories, we move on to discuss the Lagrangian which describes the standard model extended to include a LeeWick partner for each particle. The gauge sector is as before.

\section{A. The Higgs sector}

A higher derivative Lee-Wick Higgs sector was considered previously in [10]. We take the higher derivative Lagrangian for the Higgs doublet $\hat{H}$ to be

$\mathcal{L}_{\mathrm{hd}}=\left(\hat{D}_{\mu} \hat{H}\right)^{\dagger}\left(\hat{D}^{\mu} \hat{H}\right)-\frac{1}{M_{H}^{2}}\left(\hat{D}_{\mu} \hat{D}^{\mu} \hat{H}\right)^{\dagger}\left(\hat{D}_{\nu} \hat{D}^{\nu} \hat{H}\right)-V(\hat{H})$,

where the covariant derivative is given by

$$
\hat{D}_{\mu}=\partial_{\mu}+i g \hat{A}_{\mu}^{A} T^{A}+i g_{2} \hat{W}_{\mu}^{a} T^{a}+i g_{1} \hat{B}_{\mu} Y,
$$

while the potential is

$$
V(\hat{H})=\frac{\lambda}{4}\left(\hat{H}^{\dagger} \hat{H}-\frac{v^{2}}{2}\right)^{2} .
$$


We can then eliminate the higher derivative term by introducing a LW-Higgs doublet $\tilde{H}$. As before, we then diagonalize the Lagrangian by introducing the shifted field $\hat{H}=H-\tilde{H}$. To diagonalize the gauge field Lagrangian, we introduced Lee-Wick gauge bosons $\tilde{A}, \tilde{B}$, and $\tilde{W}$ as well as the usual gauge fields $A, B$, and $W$. In terms of these fields the covariant derivative is

$$
\hat{D}_{\mu}=D_{\mu}+i g \tilde{A}_{\mu}^{A} T^{A}+i g_{2} \tilde{W}_{\mu}^{a} T^{a}+i g_{1} \tilde{B}_{\mu} Y,
$$

where

$$
D_{\mu}=\partial_{\mu}+i g A_{\mu}^{A} T^{A}+i g_{2} W_{\mu}^{a} T^{a}+i g_{1} B_{\mu} Y
$$

is the usual standard model covariant derivative. We introduce the notation

$$
\tilde{\mathbf{A}}_{\mu}=g \tilde{A}_{\mu}^{A} T^{A}+g_{2} \tilde{W}_{\mu}^{a} T^{a}+g_{1} \tilde{B}_{\mu} Y
$$

for the LW-gauge bosons. The Lee-Wick form of the Higgs Lagrangian is then

$$
\begin{aligned}
\mathcal{L}= & \left(D_{\mu} H\right)^{\dagger} D^{\mu} H-\left(D_{\mu} \tilde{H}\right)^{\dagger} D^{\mu} \tilde{H}+M_{H}^{2} \tilde{H}^{\dagger} \tilde{H} \\
& -V(H, \tilde{H})+i\left(D_{\mu} H\right)^{\dagger} \tilde{\mathbf{A}}^{\mu} H-i H^{\dagger} \tilde{\mathbf{A}}_{\mu} D^{\mu} H \\
& +H^{\dagger} \tilde{\mathbf{A}}_{\mu} \tilde{\mathbf{A}}^{\mu} H-i\left(D_{\mu} \tilde{H}\right)^{\dagger} \tilde{\mathbf{A}}^{\mu} \tilde{H} \\
& +i \tilde{H}^{\dagger} \tilde{\mathbf{A}}_{\mu} D^{\mu} \tilde{H}-\tilde{H}^{\dagger} \tilde{\mathbf{A}}_{\mu} \tilde{\mathbf{A}}^{\mu} \tilde{H},
\end{aligned}
$$

where $V$ is given by the expression

$$
\begin{aligned}
V(H, \tilde{H})= & V(H-\tilde{H}) \\
= & \frac{\lambda}{4}\left(H^{\dagger} H-\frac{v^{2}}{2}\right)^{2}+\frac{\lambda}{2}\left(H^{\dagger} H-\frac{v^{2}}{2}\right) \tilde{H}^{\dagger} \tilde{H} \\
& -\frac{\lambda}{2}\left(H^{\dagger} H-\frac{v^{2}}{2}\right)\left(\tilde{H}^{\dagger} H+H^{\dagger} \tilde{H}\right)+\frac{\lambda}{4}\left[\left(H^{\dagger} \tilde{H}\right)^{2}\right. \\
& +\left(\tilde{H}^{\dagger} H\right)^{2}+\left(\tilde{H}^{\dagger} \tilde{H}\right)^{2}+2\left(H^{\dagger} \tilde{H}\right)\left(\tilde{H}^{\dagger} H\right) \\
& \left.-2\left(H^{\dagger} \tilde{H}\right)\left(\tilde{H}^{\dagger} \tilde{H}\right)-2\left(\tilde{H}^{\dagger} H\right)\left(\tilde{H}^{\dagger} \tilde{H}\right)\right]
\end{aligned}
$$

In unitary gauge, we write

$$
H=\left(\begin{array}{c}
0 \\
\frac{v+h}{\sqrt{2}}
\end{array}\right), \quad \tilde{H}=\left(\begin{array}{c}
\tilde{h}^{+} \\
\frac{\tilde{h}+i \tilde{P}}{\sqrt{2}}
\end{array}\right) .
$$

With this choice, the mass Lagrangian for the Higgs scalar, its partner, the charged LW-Higgs, and pseudoscalar LWHiggs fields is

$$
\mathcal{L}_{\text {mass }}=-\frac{\lambda}{4} v^{2}(h-\tilde{h})^{2}+\frac{M_{H}^{2}}{2}\left(\tilde{h} \tilde{h}+\tilde{P} \tilde{P}+2 \tilde{h}^{+} \tilde{h}^{-}\right) .
$$

There is mixing between the usual Higgs scalar and its partner; this mixing can be treated perturbatively. It is possible to diagonalize the mass matrices of these particles via a symplectic rotation, which preserves the diagonal form of the kinetic terms.

The Higgs vacuum expectation value induces masses for the gauge bosons. First, we focus on the mass Lagrangian for the LW-gauge bosons. In terms of the $S U(2)$ and $U(1)$ LW-gauge fields, the Lagrangian is

$$
\begin{aligned}
\mathcal{L}_{\text {mass }}= & \frac{g_{2}^{2} v^{2}}{8}\left(\tilde{W}_{\mu}^{a} \tilde{W}^{a \mu}\right)-\frac{g_{1} g_{2} v^{2}}{4} \tilde{W}_{\mu}^{3} \tilde{B}^{\mu}+\frac{g_{1}^{2} v^{2}}{8} \tilde{B}_{\mu} \tilde{B}^{\mu} \\
& -\frac{M_{1}^{2}}{2} \tilde{B}_{\mu} \tilde{B}^{\mu}-\frac{M_{2}^{2}}{2} \tilde{W}_{\mu}^{a} \tilde{W}^{a \mu} .
\end{aligned}
$$

There is mixing between the $\tilde{W}^{3}$ and $\tilde{B}$ LW-gauge fields. We can diagonalize this Lagrangian by writing

$$
\left(\begin{array}{c}
\tilde{W}^{3} \\
\tilde{B}
\end{array}\right)=\left(\begin{array}{cc}
\cos \phi & \sin \phi \\
-\sin \phi & \cos \phi
\end{array}\right)\left(\begin{array}{c}
\tilde{U} \\
\tilde{V}
\end{array}\right)
$$

where the mixing angle is given by

$$
\tan 2 \phi=\frac{g_{1} g_{2} v^{2}}{2}\left(M_{1}^{2}-M_{2}^{2}+\left(g_{2}^{2}-g_{1}^{2}\right) \frac{v^{2}}{4}\right)^{-1} .
$$

We expect that $M_{1,2}$ lie in the $\mathrm{TeV}$ range, so that $\phi$ is a small angle.

There is also mixing between the gauge fields and the LW-gauge fields. We will treat this mixing perturbatively. The Lagrangian describing this mixing is

$$
\begin{aligned}
\mathcal{L}_{\text {mix }}= & M_{W}^{2}\left(W_{\mu}^{+} \tilde{W}^{-\mu}+\tilde{W}_{\mu}^{+} W^{-\mu}\right) \\
& +M_{Z}^{2} Z_{\mu}\left(\cos \theta_{W} \tilde{W}^{3 \mu}-\sin \theta_{W} \tilde{B}^{\mu}\right),
\end{aligned}
$$

where $\theta_{W}$ is the Weinberg angle and $M_{W}, M_{Z}$ are the usual tree-level standard model masses for the $W$ and $Z$ gauge bosons. One consequence of the mixing is that there is a tree-level correction to the electroweak $\rho$ parameter

$$
\Delta \rho=\rho-1=-\frac{\sin ^{2} \theta_{W} M_{Z}^{2}}{M_{1}^{2}} .
$$

The current experimental constraint on this parameter is $|\Delta \rho| \lesssim 10^{-3}[14]$ which leads to $M_{1} \gtrsim 1 \mathrm{TeV}$.

\section{B. Fermion kinetic terms}

For simplicity, we discuss explicitly the case of a single left-handed quark doublet $Q_{L}$. It is straightforward to generalize this work to the other representations, and to include generation indices.

The higher derivative theory is

$$
\mathcal{L}_{\mathrm{hd}}=\overline{\hat{Q}}_{L} i \hat{\not D} \hat{Q}_{L}+\frac{1}{M_{Q}^{2}} \overline{\hat{Q}}_{L} i \hat{\not D} \hat{\not D} \hat{\not D} \hat{Q}_{L} .
$$

Naive power counting of the possible divergences in this higher derivative theory shows that there are potential quadratic divergences in one-loop graphs containing two external gauge bosons and a fermionic loop. However, gauge invariance forces these graphs to be proportional to two powers of the external momentum so that the graphs are only logarithmically divergent. In this case, this cancellation is most easily understood in the LW description of the theory, which we now construct.

We eliminate the higher derivative term by introducing LW-quark doublets $\tilde{Q}_{L}, \tilde{Q}_{R}^{\prime}$ which form a real representa- 
tion of the gauge groups. The Lagrangian in this formulation becomes

$$
\begin{aligned}
\mathcal{L}= & \overline{\hat{Q}}_{L} i \hat{\not} \hat{Q}_{L}+M_{Q}\left(\overline{\tilde{Q}}_{L} \tilde{Q}_{R}^{\prime}+\overline{\tilde{Q}}_{R}^{\prime} \tilde{Q}_{L}\right)+\overline{\tilde{Q}}_{L} i \hat{\not} \hat{Q}_{L} \\
& +\overline{\hat{Q}}_{L} i \hat{\not D} \tilde{Q}_{L}-\overline{\tilde{Q}}_{R}^{\prime} i \hat{\not D} \tilde{Q}_{R}^{\prime} .
\end{aligned}
$$

Eliminating the LW fermions with their equations of motion

$$
\tilde{Q}_{R}^{\prime}=-\frac{i \hat{\not D}}{M_{Q}} \hat{Q}_{L}, \quad \tilde{Q}_{L}=\frac{\hat{\not D} \hat{\not D}}{M_{Q}^{2}} \hat{Q}_{L},
$$

reproduces the higher derivative Lagrangian, Eq. (51).

To diagonalize the kinetic terms, we introduce the shift $\hat{Q}_{L}=Q_{L}-\tilde{Q}_{L}$, and the Lagrangian becomes

$$
\begin{aligned}
\mathcal{L}= & \bar{Q}_{L} i \not D Q_{L}-\overline{\tilde{Q}}_{L} i \not D \tilde{Q}_{L}-\overline{\tilde{Q}}_{R}^{\prime} i \not D \tilde{Q}_{R}^{\prime} \\
& +M_{Q}\left(\overline{\tilde{Q}}_{L} \tilde{Q}_{R}^{\prime}+\overline{\tilde{Q}}_{R}^{\prime} \tilde{Q}_{L}\right)-\bar{Q}_{L} \gamma_{\mu} \tilde{\mathbf{A}}^{\mu} Q_{L} \\
& +\overline{\tilde{Q}}_{L} \gamma_{\mu} \tilde{\mathbf{A}}^{\mu} \tilde{Q}_{L}+\overline{\tilde{Q}}_{R}^{\prime} \gamma_{\mu} \tilde{\mathbf{A}}^{\mu} \tilde{Q}_{R}^{\prime} .
\end{aligned}
$$

Note that $\tilde{Q}_{L}$ and $\tilde{Q}_{R}^{\prime}$ combine into a single Dirac spinor of mass $M_{Q}$.

Now let us return to the issue of potential quadratic divergences in the theory. Inspection of the Lagrangian, Eq. (54), shows that the only one-loop graphs involving fermionic loops are the graphs of Fig. 4. Figure 4(a) is a one-loop correction to the gauge boson propagator, and consequently is proportional to $p^{2}$, where $p$ is the momentum flowing into the graph. Thus, the graph is logarithmically divergent, as is well known. Figure 4(b) is a one-loop correction to the LW-gauge boson propagator. One might think that this graph could introduce a quadratic divergence of the LW-gauge boson mass. However, the vertices between the fermions and the gauge bosons are equal to the vertices between the fermions and the LW-gauge bosons, as can be seen in Eq. (54). Thus, Fig. 4(b) is logarithmically divergent. Higher loop graphs in the theory are at most logarithmically divergent by power counting.

\section{Fermion Yukawa interactions}

To simplify the discussion in this section, we will neglect neutrino masses. In the higher derivative formulation, the fermion Yukawas are

$$
\mathcal{L}_{Y}=g_{u}^{i j} \overline{\hat{u}}^{i}{ }_{R} \hat{H} \epsilon \hat{Q}_{L}^{j}-g_{d}^{i j} \overline{\hat{d}}_{R}^{i} \hat{H}^{\dagger} \hat{Q}_{L}^{j}-g_{e}^{i j} \overline{\hat{e}}_{R}^{i} \hat{H}^{\dagger} \hat{L}_{L}^{j}+\text { H.c., }
$$

where repeated flavor indices are summed. In the formu-

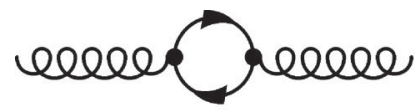

(a)

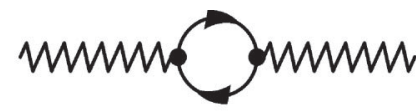

(b)
FIG. 4. One-loop graphs involving fermions which are potentially quadratically divergent. The solid lines represent fermion propagators while the curly and zigzag lines represent gauge bosons and LW-gauge bosons, respectively. lation of the theory in which there are no higher derivatives, and in which the kinetic terms are diagonal, this becomes

$$
\begin{aligned}
\mathcal{L}_{Y}= & g_{u}^{i j}\left(\bar{u}_{R}^{i}-\overline{\tilde{u}}_{R}^{i}\right)(H-\tilde{H}) \epsilon\left(Q_{L}^{j}-\tilde{Q}_{L}^{j}\right) \\
& -g_{d}^{i j}\left(\bar{d}_{R}^{i}-\overline{\tilde{d}}_{R}^{i}\right)\left(H^{\dagger}-\tilde{H}^{\dagger}\right)\left(Q_{L}^{j}-\tilde{Q}_{L}^{j}\right) \\
& -g_{e}^{i j}\left(\bar{e}^{i}{ }_{R}-\overline{\tilde{e}}_{R}^{i}\right)\left(H^{\dagger}-\tilde{H}^{\dagger}\right)\left(L_{L}^{j}-\tilde{L}_{L}^{j}\right)+\text { H.c. }
\end{aligned}
$$

The presence of the LW fields in this equation improves the degree of convergence at one loop. For example, consider a one-loop correction to the Higgs two point function coming from the first term of Eq. (56). Various degrees of freedom can propagate in the loop: the $u_{R}$ and $Q_{L}$ quarks, and also the $\tilde{u}_{R}$ and $\tilde{Q}_{L} \mathrm{LW}$ quarks. The presence of the LW quarks cancels the quadratic divergence in the loop with only the quarks. The sum of these four graphs reproduces the result one would find by computing the corresponding correction in the higher derivative formulation of the theory, Eq. (55).

To simplify the flavor structure of the theory, we adopt the principle of minimal flavor violation ${ }^{8}$ [16]. This forces all LW fermions in the same representation of the gauge group have the same mass. Now the Yukawas can be diagonalized in the standard fashion. For notational brevity, we choose to use the same symbol for the weak and mass eigenstates. In terms of the mass eigenstate fields, ${ }^{9}$

$$
\begin{aligned}
\mathcal{L}_{Y}= & \frac{\sqrt{2}}{v} \sum_{i}\left[m_{u}^{i}\left(\bar{u}^{i}{ }_{R}-\overline{\tilde{u}}_{R}^{i}\right)(H-\tilde{H}) \epsilon\left(Q_{L}^{i}-\tilde{Q}_{L}^{i}\right)\right. \\
& -m_{d}^{i}\left(\bar{d}^{i}{ }_{R}-\overline{\tilde{d}}_{R}^{i}\right)\left(H^{\dagger}-\tilde{H}^{\dagger}\right)\left(Q_{L}^{i}-\tilde{Q}_{L}^{i}\right) \\
& \left.-m_{e}^{i}\left(\bar{e}^{i}{ }_{R}-\overline{\tilde{e}}^{i}{ }_{R}\right)\left(H^{\dagger}-\tilde{H}^{\dagger}\right)\left(L_{L}^{i}-\tilde{L}_{L}^{i}\right)+\text { H.c. }\right],
\end{aligned}
$$

where

$$
Q_{L}=\left(\begin{array}{c}
u_{L} \\
V d_{L}
\end{array}\right), \quad \tilde{Q}_{L}=\left(\begin{array}{c}
\tilde{u}_{L} \\
V \tilde{d}_{L}
\end{array}\right), \quad \tilde{Q}_{R}^{\prime}=\left(\begin{array}{c}
\tilde{u}_{R}^{\prime} \\
V \tilde{d}_{R}^{\prime}
\end{array}\right)
$$

Here $V$ is the usual CKM matrix. The LW fermions decay via the Yukawa interactions; for example, $\tilde{\nu}_{e} \rightarrow e^{-} \tilde{h}^{+} \rightarrow$ $e^{-} t \bar{b}$. LW-gauge bosons can decay to pairs of ordinary fermions. All the heavy LW particles decay in this theory, so the only sources of missing energy in collider experiments are the usual standard model neutrinos.

\footnotetext{
${ }^{8}$ Since completion of this work, it has been shown that flavorchanging neutral currents are suppressed in the Lee-Wick standard model without assuming the MFV hypothesis [15].

${ }^{9}$ They are mass eigenstate fields when mixing between the normal and LW fields is neglected. This mixing can be treated as a perturbation.
} 


\section{CONCLUSIONS}

In this paper we have developed an extension of the minimal standard model that is free of quadratic divergences. It is based on the work of Lee and Wick who constructed a finite version of QED by associating the regulator propagator in Pauli-Villars with a physical degree of freedom. Our model is a higher derivative theory and as such contains propagators with wrong sign residues about the new poles. Lee and Wick, and Cutkosky et al. provide a prescription for handling this issue. The LW particles associated with these new poles are not in the spectrum, but instead decay to ordinary degrees of freedom. Their resummed propagators do not satisfy the usual analyticity properties since the poles are on the physical sheet. Lee and Wick (see also Cutkosky et al.) propose deforming integration contours in Feynman diagrams so that there is no catastrophic exponential growth as time increases. This amounts to a future boundary condition and so LW theories violate the usual causal conditions. While the Lee-Wick interpretation is peculiar it seems to be consistent, ${ }^{10}$ at least in perturbation theory, and predictions for physical observables can be made order by order in perturbation theory.

\footnotetext{
${ }^{10}$ Unitarity has not been demonstrated to all orders in perturbation theory and this remains one of the important outstanding issues for the Lee-Wick standard model.
}

Since the extension of the standard model presented here is free of quadratic divergences it solves the hierarchy problem. Our theory contains one new parameter, the mass of the LW- partner, for each field. We reduced the number of parameters by imposing minimal flavor violation to simplify the flavor structure of the theory. To make the physical interpretation clearer and the calculations easier we introduced auxiliary LW fields. The Lagrangian written in terms of these fields does not contain any higher derivative terms. When the LW fields are integrated out the higher derivative theory is recovered.

This paper focused on the structure of the Lagrange density for the Lee-Wick extension of the standard model. We constructed the Lagrange density, examined the divergence structure and showed how to introduce auxiliary fields to clarify the physical interpretation. For the future, a more extensive discussion of the phenomenology of the theory including its implications for LHC physics is appropriate.

\section{ACKNOWLEDGMENTS}

D. O.C. would like to thank Stephen Adler for a helpful discussion and for pointing out a useful reference. The work of B.G. was supported in part by the U.S. Department of Energy under Contract No. DE-FG0397ER40546, while the work of D. O.C. and M. B. W. was supported in part by the U.S. Department of Energy under Contract No. DE-FG03-92ER40701.
[1] T. D. Lee and G. C. Wick, Nucl. Phys. B9, 209 (1969).

[2] T.D. Lee and G. C. Wick, Phys. Rev. D 2, 1033 (1970).

[3] R.E. Cutkosky, P. V. Landshoff, D. I. Olive, and J.C. Polkinghorne, Nucl. Phys. B12, 281 (1969).

[4] N. Nakanishi, Phys. Rev. D 3, 811 (1971).

[5] T. D. Lee and G. C. Wick, Phys. Rev. D 3, 1046 (1971).

[6] N. Nakanishi, Phys. Rev. D 3, 1343 (1971).

[7] S. Coleman, in Subnuclear Phenomena, edited by A. Zichichi (Academic, New York, 1970), p. 282.

[8] S. W. Hawking and T. Hertog, Phys. Rev. D 65, 103515 (2002).

[9] D. G. Boulware and D. J. Gross, Nucl. Phys. B233, 1 (1984).

[10] K. Jansen, J. Kuti, and C. Liu, Phys. Lett. B 309, 119
(1993); 309, 127 (1993).

[11] I. Antoniadis and E. T. Tomboulis, Phys. Rev. D 33, 2756 (1986).

[12] J. Pestieau and P. Roy, Phys. Rev. Lett. 23, 349 (1969).

[13] D. Evens, J. W. Moffat, G. Kleppe, and R. P. Woodard, Phys. Rev. D 43, 499 (1991); J. W. Moffat, arXiv:hep-ph/ 0003171.

[14] W. M. Yao et al. (Particle Data Group), J. Phys. G 33, 1 (2006).

[15] T. R. Dulaney and M. B. Wise, arXiv:0708.0567.

[16] R.S. Chivukula and H. Georgi, Phys. Lett. B 188, 99 (1987); L. J. Hall and L. Randall, Phys. Rev. Lett. 65, 2939 (1990); G. D’Ambrosio, G. F. Giudice, G. Isidori, and A. Strumia, Nucl. Phys. B645, 155 (2002). 